\title{
Assessment of Criteria Regarding CpG Islands of Their Role as Gene Marker
}

\author{
Haewon Kim, Jongjun Lee, Seonghui Yu, Junhyung Bae, Yeonho Jung, and Taeseon Yoon
}

\begin{abstract}
CpG islands are clusters of CG-rich DNA sequences, approximately half of which exist in 5 ' region of human housekeeping genes. Using various AI algorithms, previous studies classified CpG islands based on the criteria: length $\geq 200 \mathrm{bp}, \% \mathrm{GC} \geq 50 \%$, and ObsCpG(Observed CpG)/ExpCpG(Expected CpG) $\geq 0.60$. They showed that CpG islands overlap the promoter of all human housekeeping genes and over half of all tissue-specific genes.

Using CpGIE(CpG Island Explorer), we evaluated suggested criteria for the search of CpG island of human gene chromosome 18, 19, 20. Manipulating three different criteria: length, \%GC, and ObsCpG/ExpCpG rate, we found most appropriate criteria for $\mathrm{CpG}$ detection. One of the most important processes of our assessment is to decide whether the new criteria effectively exclude $\mathrm{Alu}$ repeats. Moreover, by assorting association type: promoter-relation, within-relation, end-relation, we confirmed that $\mathrm{CpG}$ islands defined by the new criteria showed better function as gene markers.
\end{abstract}

Index Terms-Bioinformatics, CpG island, gene marker.

\section{INTRODUCTION}

In DNA sequence of human gene, CG appears less frequently than other dinucleotide sequences such as GG, GA, GT, and GC. However, in the 5' end of gene, the frequency rises up to the expected value. The clusters of the dinucleotide observed in this part of gene are called $\mathrm{CpG}$ islands. There are about $45,000 \mathrm{CpG}$ islands in the human gene. These $\mathrm{CpG}$ islands are considered to be crucial in cell differentiation and regulation of gene expression. [1] As most $\mathrm{CpG}$ islands are located near the promoters, these $\mathrm{CpG}$ islands are employed as gene markers which indicate where the promoters and first exons of human genomes are. [2] Thus, plotting $\mathrm{CpG}$ islands in human gene can show the association between themselves and promoters, and, furthermore, the effect of $\mathrm{CpG}$ dinucleotide on human gene expression.

Criteria employed to identify $\mathrm{CpG}$ islands have been improved over time. In the primary studies, criteria for a $\mathrm{CpG}$ island were a DNA sequence longer than $200 \mathrm{bp} ; \% \mathrm{GC} \geq$ 50\%; CpGobs(observed)/exp(expected) $\geq 0.6$ [3].

CpGobs(observed)/exp(expected) means the number of $\mathrm{CpG}$ divided by the number of $\mathrm{C} \times$ the number of $\mathrm{G} \times \mathrm{N}$. ( $\mathrm{N}$ is the total number of nucleotides in the sequence which is analyzed) Recently, more strict criteria are developed. Takai and Jones proposed criteria of length longer than 200

Manuscript received November 10, 2013; revised January 10, 2014.

The authors are with Hankuk Academy of Foreign Studies, Wangsan-ri, Mohyeon-myeon, Cheoin-gu, Yongin-si, Gyeonggi-do, Korea (e-mail: janny4615@naver.com, 1jj960819@gmail.com, seonghui12@gmail.com, hjweebee1@naver.com, gwi03186@ naver.com). bp; \%GC $\geq 55 \%$; CpGobs/exp $\geq 0.65$ [4] Despite concerns that new criteria could also exclude already identified $\mathrm{CpG}$ islands, reports showed that the proportion of genes with $\mathrm{CpG}$ islands was almost the same under the old and the generally accepted criteria [5].

Previous studies employed classification methods such as AI algorithms, neural networks. The classification became more sophisticated. The result showed an improvement from the previous studies. Also, the experiment was taken on homo sapiens chromosome 18, 19, 20.

\section{PURPOSE}

\section{A. Expected Results and Purpose of Experiment}

Our goal is to predict housekeeping genes associated with CpGisland effectively and accurately. We tried to make new criteria which can precisely predict the association between genes and $\mathrm{CpG}$ islands. With a computer program counting $\mathrm{CpG}$ islands on different parts of genes, we expected to find new criteria that can define promoter-associated $\mathrm{CpG}$ islands more effectively.

\section{MEANS OF EXPERIMENT}

\section{A. Procedure of Equipment}

\section{1) CpGIE (CpG island explorer)}

The equipment used for our experiment is $\mathrm{CpGIE}(\mathrm{CpG}$ Island Explorer). CpGIE is a java program developed by Yong Wang et al. [6] It is a tool for CpG island searching. It has interface panel in which criteria used for $\mathrm{CpG}$ island search can be manipulated. Sequences can be loaded on the program, and the program graphically demonstrates $\mathrm{CpG}$ islands on the genes. Compared with other programs, it showed better performance than other prorams such as CpGPlot, and CpGProD.[6]

\section{2) Algoritm}

To search for $\mathrm{CpG}$ island, we used CPGIE-a java program for $\mathrm{CpG}$ island analysis, developed by Takai and Jones [4]. The main processes to manipulate CPGIE are followed:

\section{a) Edit the input data \\ b) Sorting the initial $C p G$ islands out}

- Set up the criteria, In this study, we searched CpG islands according to three different criteria: length $\geq 500 \mathrm{bp}$, $\mathrm{G}+\mathrm{C}$ content $\geq 60 \%$, and $\mathrm{CpG}$, o/e ratio $\geq 0.70$ (newly suggested criteria 1) and length $\geq 500 \mathrm{bp}, \mathrm{G}+\mathrm{C}$ content $\geq 60 \%$, and $\mathrm{CpG}$, o/e ratio $\geq 0.60$ (newly suggested criteria 2 ) and length $\geq 500 \mathrm{bp}, \mathrm{G}+\mathrm{C}$ content $\geq 55 \%$, and $\mathrm{CpG}$, o/e ratio $\geq$ 0.65 (generally accepted criteria by previous studies) . 
- Considering the DNA sequence which was inputted by the first input steps, minimize the length of window. Before identify the satisfying criteria, a moving window have to adjust at least ( $\mathrm{CpG}$ o/e ratio) * (minimum length) $/ 16 \mathrm{CpG}$ dinucleotides. This procedure will eliminate mathematical $\mathrm{CpG}$ islands which were caused by an enhanced component proportion ratio of $\mathrm{G}$ over $\mathrm{C}$, or the transposed [4]

- The beginning points are marked if some regions in a window were verified as a $\mathrm{CpG}$ islands. Then, at intervals of $10 \mathrm{nt}$, the window moves ahead to find the first region in the window that is not a $\mathrm{CpG}$ island. It is also marked that the stop position of the $\mathrm{CpG}$ islands are in the last window.

- If there is a sequence that fulfill the criteria in the middle of marked area (between start site and stop site), it is classified as a initial $\mathrm{CpG}$ island. Otherwise, until it meets satisfying criteria, both ends of the sequence will be cut at the intervals of $1 \mathrm{nt}$ simultaneously.

- The window keeps moving at interval of 1 nt. To reiterate the proposed processes (ii, iii, iv) enable CpGIE to search all the initial $\mathrm{CpG}$ islands in their location order.

\section{c) Integrate the initial $\mathrm{CpG}$ islands}

The recorded initial $\mathrm{CpG}$ islands are mostly superimposed due to the subtle moving steps of previous steps. Draw a comparison of the end site of an initial $\mathrm{CpG}$ island and the start site of the circumjacent backward $\mathrm{CpG}$ island, and assemble the superimposing and close-spaced $(<100 \mathrm{nt}$ in distance) initial $\mathrm{CpG}$ islands in a group. To create a final $\mathrm{CpG}$ island, initial $\mathrm{CpG}$ islands in the equal group will be employed.

The location that the $\mathrm{CpG}$ islands possess in the DNA sequence-between the start position and stop position of the initial $\mathrm{CpG}$ island- is decided by the criteria. If there are cutting processes, it will keep performing to find the final $\mathrm{CpG}$ islands in the region.

Although the cutting process of the sequence in the region was performed, no final $\mathrm{CpG}$ island is discovered in some cases. In these respects, the initial $\mathrm{CpG}$ islands usually in the middle of the region show the final island in this group.

\section{3) Datasets}

We excerpted all the information about the contigs in human chromosomes from NCBI (http://www.ncbi.nlm.nih.gov). The contigs in the chromosome 18, 19, 20 we used in this study were: NT_010966.14, NT_010895.14, NT_011109.16, NT_077812.2, NT011362.10, NT_011333.6, NT_025215.4. Information about the genes and contigs with their start and end sites, transcription orientation and evidence code used in this study was acquired from the NCBI.

\section{4) Connection between CpG Island and Genes}

Using CpGIE, we searched $\mathrm{CpG}$ islands in chromosomes 18,19 , and 20 based on the generally accepted criteria (by previous studies): length $\geq 500 \mathrm{bp}, \% \mathrm{GC}$ content $\geq 55 \%$, and $\mathrm{CpG}, \mathrm{o} / \mathrm{e}$ ratio $\geq 0.65$. And repeat the process on the basis of newly suggested criteria: length $\geq 500 \mathrm{bp}, \mathrm{G}+\mathrm{C}$ content $\geq$ $60 \%$, and $\mathrm{CpG}, \mathrm{o} / \mathrm{e}$ ratio $\geq 0.70$ (new criteria 1 ) and length $\geq$ $500 \mathrm{bp}, \mathrm{G}+\mathrm{C}$ content $\geq 60 \%$, and $\mathrm{CpG}$, o/e ratio $\geq 0.60$ (new criteria 2). Similar to the genes in the contigs, the $\mathrm{CpG}$ islands appeared depending on their start site and end site location.

According to suggested criteria and generally accepted criteria for the search of $\mathrm{CpG}$ islands, we marks the genes, $\mathrm{CpG}$ islands on the contigs. We categorized the connections between $\mathrm{CpG}$ islands and genes into promoter relation, within relation and end relation regarding its association.

To visualize the correlation between $\mathrm{CpG}$ island location and gene location, we made a graph with sequence NT_025215.4 in chromosome 20. We use Microsoft Excel program to array nucleotide sequences, and marking them. In Microsoft Excel program, base pairs are arrayed 70 pairs per row, so we make chart to display quotient and remainder of start and end point when they are divided by 70 . Also, we set 70 base pairs as one window, and transfer windows with 70 pairs each. We got CG percentage, marked gene and marked $\mathrm{CpG}$ islands by each window. Then we could get the graph by marking $\mathrm{CpG}$ islands and Gene induced through each other criteria.

\section{RESUlTS AND DISCUSSION}

\section{A. New Criteria Are More Effective in Finding $\mathrm{CpG}$ Islands Related to Genes}

The role of $\mathrm{CpG}$ islands as gene markers are assessed by their relativity to genes. There are simply two parts in chromosomes. One of them is gene section, and the other one is the part that remains after excluding gene section from the chromosome. By using CpGIE as mentioned before, the total number of $\mathrm{CpG}$ islands in the whole contig and the number of $\mathrm{CpG}$ islands that is related to genes were checked.

This observation was progressed based on two newly suggested criteria which are criteria 1 : length $\geq 500 \mathrm{bp}, \mathrm{G}+\mathrm{C}$ content $\geq 60 \%$, and $\mathrm{CpG}, \mathrm{o} / \mathrm{e}$ ratio $\geq 0.60$, criteria 2 : length $\geq$ $500 \mathrm{bp}, \mathrm{G}+\mathrm{C}$ content $\geq 60 \%$, and $\mathrm{CpG}$, o/e ratio $\geq 0.70$ and one generally accepted criteria : length $\geq 500 \mathrm{bp}, \mathrm{G}+\mathrm{C}$ content $\geq 55 \%$, and $\mathrm{CpG}$, o/e ratio $\geq 0.65$, and this will be referred to as established criteria. [6] The $\mathrm{CpG}$ islands related to genes include promoter-related, within-related, and end-related CpG islands (see Table I-IV).

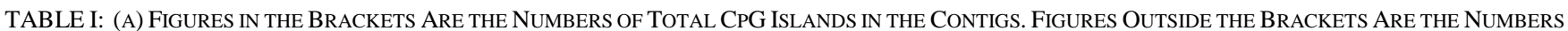
OF CPG ISLANDS THAT ARE RELATED TO GENES

\begin{tabular}{llll} 
& \multicolumn{2}{c}{ OF CPG ISLANDS THAT ARE RELATED TO GENES } & Established criteria \\
NT_010859.14 & New criteria 1 & New criteria 2 & $131(178)$ \\
NT_010966.14 & $96(158)$ & $93(120)$ & $167(220)$ \\
NT_011109.16 & $130(171)$ & $129(143)$ & $1067(1329)$ \\
NT_077812.2 & $995(1160)$ & $759(945)$ & $73(78)$ \\
NT_011333.6 & $70(72)$ & $54(56)$ & $163(176)$ \\
NT_011362.10 & $161(167)$ & $104(106)$ & $180(536)$ \\
NT_025215.4 & $173(454)$ & $134(339)$ & $1(7)$ \\
\hline
\end{tabular}


TABLE I: (B) GENE-RELATION RATE OF THREE DIFFERENT CRITERIA

\begin{tabular}{|c|c|c|c|}
\hline & New criteria 1 & New criteria 2 & Established criteria \\
\hline NT_010859.14 & 0.620 & 0.775 & 0.736 \\
\hline NT_010966.14 & 0.760 & 0.902 & 0.759 \\
\hline NT_011109.16 & 0.858 & 0.803 & 0.803 \\
\hline NT_077812.2 & 0.972 & 0.964 & 0.936 \\
\hline NT_011333.6 & 0.964 & 0.981 & 0.926 \\
\hline NT_011362.10 & 0.381 & 0.395 & 0.336 \\
\hline NT_025215.4 & 0.143 & 0.000 & 0.143 \\
\hline
\end{tabular}

TABLE II: The Number OF PROMOTER-RELATED, WITHIN-RELATED, END-RELATED CPG ISLANDS, DETERMINED BY NEW CRITERIA 1

\begin{tabular}{c|c|c|c|c|c}
\hline Chromosome & Contig & Promoter & Within & End & Promoter Relation Rate \\
\hline 18 & NT_010859.14 & 56 & 39 & 1 & 58.333 \\
\hline 18 & NT_010966.14 & 70 & 60 & 0 & 53.846 \\
\hline 19 & NT_011109.16 & 310 & 635 & 50 & 31.156 \\
\hline 19 & NT_077812.2 & 24 & 42 & 4 & 34.286 \\
\hline 20 & NT_011333.6 & 41 & 119 & 1 & 25.466 \\
\hline 20 & NT_011362.10 & 31 & 68 & 2 & 30.693 \\
\hline 20 & NT_025215.4 & 0 & 1 & 0 & 0 \\
\hline & * Criteria are \%GC 60\%, O/E 0.60, length 500 bp \\
\hline
\end{tabular}

TABLE III: The NuMBER OF PROMOTER-RELATED, WITHIN-RELATED, END-RELATED CPG ISLANDS, DETERMINED BY NEW CRITERIA 2

\begin{tabular}{c|c|c|c|c|c}
\hline Chromosome & Contig & Promoter & Within & End & Promoter Relation Rate \\
\hline 18 & NT_010859.14 & 54 & 38 & 1 & 58.065 \\
\hline 18 & NT_010966.14 & 71 & 57 & 1 & 55.039 \\
\hline 19 & NT_011109.16 & 240 & 497 & 22 & 31.621 \\
\hline 19 & NT_077812.2 & 23 & 29 & 2 & 42.593 \\
\hline 20 & NT_011333.6 & 35 & 68 & 1 & 33.654 \\
\hline 20 & NT_011362.10 & 71 & 62 & 1 & 52.985 \\
\hline 20 & NT_025215.4 & 0 & 0 & 0 & 0 \\
\hline & * Criteria are \%GC 60\%, O/E 0.70, length 500 bp \\
\hline
\end{tabular}

TABLE IV: THE NuMBER OF PROMOTER-RELATED, WITHIN-RELATED, END-RELATED CPG ISLANDS, DETERMINED BY ESTABLISHED CRITERIA

\begin{tabular}{c|c|c|c|c|c}
\hline Chromosome & Contig & Promoter & Within & End & Promoter Relation Rate \\
\hline 18 & NT_010859.14 & 63 & 66 & 2 & 48.092 \\
\hline 18 & NT_010966.14 & 85 & 81 & 1 & 50.898 \\
\hline 19 & NT_011109.16 & 379 & 654 & 34 & 35.520 \\
\hline 19 & NT_077812.2 & 26 & 41 & 6 & 35.616 \\
\hline 20 & NT_011333.6 & 39 & 120 & 4 & 23.926 \\
\hline 20 & NT_011362.10 & 87 & 90 & 3 & 49.333 \\
\hline 20 & NT_025215.4 & 1 & 0 & 0 & 100.000 \\
\hline
\end{tabular}

The role of $\mathrm{CpG}$ islands as gene markers is especially highlighted because $\mathrm{CpG}$ islands are highly related to the promoter section of genes [5]. In other words, the rate of promoter-related $\mathrm{CpG}$ islands is outstanding, compared to other types of gene-related $\mathrm{CpG}$ islands. The number of different types of gene-related $\mathrm{CpG}$ islands was counted.

Even if the number of newly defined $\mathrm{CpG}$ islands dropped, it is shown that new criteria are more efficient gene markers, assessed by two ways. First, gene relation rate ((The number of gene-related $\mathrm{CpG}$ islands)/(The number of total $\mathrm{CpG}$ islands in the whole contigs)) arose dramatically in two new criteria. Since one common difference of two new criteria with established criteria is $\mathrm{G}+\mathrm{C}$ content, which is more stringent than the established criteria, it is expected that higher $\mathrm{G}+\mathrm{C}$ content contributes to more efficient gene marking by using $\mathrm{CpG}$ islands.

Second, promoter relation rate ((The number of promoter-related $\mathrm{CpG}$ islands)/(The number of total $\mathrm{CpG}$ islands in the whole contigs)) arose dramatically in new criteria. It was especially higher in new criteria 2 , as it was showed in NT_011109.16 and NT_011255.14, NT_077812.2. This tendency can be contributed to two factors. First, within-related and end-related $\mathrm{CpG}$ islands might have lower $\mathrm{G}+\mathrm{C}$ content than promoter-related $\mathrm{CpG}$ islands, as promoter-related $\mathrm{CpG}$ islands better satisfied both new criteria, whose only common difference is the rise in $\mathrm{G}+\mathrm{C}$ content criteria. Also, new criteria 2 might show better promoter relation rate, owing to higher o/e rate. According to previous studies, higher $\mathrm{G}+\mathrm{C}$ content and o/e ratio are effective in filtering $A l u$ repeats [4]. Thus, more stringent $\mathrm{G}+\mathrm{C}$ content and o/e ratio criteria might contribute to new criteria becoming better criteria of $\mathrm{CpG}$ islands as gene markers.

Thus, gene relation rate and promoter relation rate proves that two new criteria are better tools of detecting gene-promoter section by using $\mathrm{CpG}$ islands.

\section{B. Comparision of New Criteria 1 and New Criteria 2 Regarding CpG Islands' Role as Gene Markers}

Comparing new criteria with established criteria using Gene relation rate and Promoter relation rate, we conclude that new criteria 2 shows better effectiveness than new criteria 1 in predicting $\mathrm{CpG}$ islands which function as gene markers. Higher gene relation rate and promoter relation rate tells that new criteria 2 are better criteria. New criteria 2 have higher o/e rate. Higher o/e rate has an effect on criteria's function as gene marker. It is especially well shown from higher promoter relation rate. This noticeably improved effectiveness is thought to be a result of higher o/e rate, which is reported to exclude Alu repeats well [4], [5]. Because Alu repeats are often miscounted as $\mathrm{CpG}$ islands that are associated with genes, this advance in excluding $A l u$ repeats shows clear difference between two criteria as their role as gene markers (see Table V-VII and Fig. 1- Fig. 4). 
TABLE V: ESTABLISHED CRITERIA

\begin{tabular}{c|c|c|c|c|c|c|c|c}
\hline Start & End & GC\% & O/E(CpG) & Length & start int & $\begin{array}{c}\text { start } \\
\text { mod }\end{array}$ & end int & $\begin{array}{c}\text { end } \\
\text { mod }\end{array}$ \\
\hline 96587 & 97316 & 61.6 & 0.65 & 730 & 1379 & 57 & 1390 & 16 \\
\hline 98900 & 99761 & 62.6 & 0.65 & 862 & 1412 & 60 & 1425 & 11 \\
\hline 104520 & 105067 & 55 & 0.68 & 548 & 1493 & 10 & 1500 & 67 \\
\hline 105571 & 106079 & 55.1 & 0.65 & 509 & 1508 & 11 & 1515 & 29 \\
\hline 115183 & 115734 & 63.8 & 0.65 & 552 & 1645 & 33 & 1653 & 24 \\
\hline 131357 & 132441 & 61.6 & 0.66 & 1085 & 1876 & 37 & 1892 & 1 \\
\hline 191388 & 192814 & 61.8 & 0.69 & 1427 & 2734 & 8 & 2754 & 34 \\
\hline
\end{tabular}

TABLE VI: NEW CRITERIA 1

\begin{tabular}{c|c|c|c|c|c|c|c|c}
\hline Start & End & GC\% & $\begin{array}{c}\text { O/E(Cp } \\
\text { G) }\end{array}$ & Length & start int & $\begin{array}{c}\text { start } \\
\text { mod }\end{array}$ & end int & $\begin{array}{c}\text { end } \\
\text { mod }\end{array}$ \\
\hline 96493 & 97332 & 60.8 & 0.61 & 840 & 1378 & 33 & 1390 & 32 \\
\hline 98724 & 99943 & 61.4 & 0.65 & 1220 & 1410 & 24 & 1427 & 53 \\
\hline 104713 & 105251 & 60 & 0.6 & 539 & 1495 & 63 & 1503 & 41 \\
\hline 106752 & 107264 & 73 & 0.6 & 513 & 1525 & 2 & 1532 & 24 \\
\hline 115201 & 115869 & 61.8 & 0.6 & 669 & 1645 & 51 & 1655 & 19 \\
\hline 131144 & 132440 & 61.5 & 0.62 & 1297 & 1873 & 34 & 1892 & 0 \\
\hline 191558 & 192913 & 61.8 & 0.69 & 1356 & 2736 & 38 & 2755 & 63 \\
\hline
\end{tabular}

TABLE VII: NEW CRITERIA 2

\begin{tabular}{c|c|c|c|c|c|c|c|c}
\hline Start & End & GC\% & $\begin{array}{c}\text { O/E(Cp } \\
\text { G) }\end{array}$ & Length & start int & $\begin{array}{c}\text { start } \\
\text { mod }\end{array}$ & end int & $\begin{array}{c}\text { end } \\
\text { mod }\end{array}$ \\
\hline 96671 & 97305 & 61.5 & 0.7 & 635 & 1381 & 1 & 1390 & 5 \\
\hline 98909 & 99698 & 63.5 & 0.7 & 790 & 1412 & 69 & 1424 & 18 \\
\hline 115084 & 115593 & 60 & 0.71 & 510 & 1644 & 4 & 1651 & 23 \\
\hline 131468 & 132381 & 61.4 & 0.7 & 914 & 1878 & 8 & 1891 & 11 \\
\hline 191558 & 192555 & 62.5 & 0.71 & 998 & 2736 & 38 & 2750 & 55 \\
\hline
\end{tabular}

\section{Percetage(\%)}

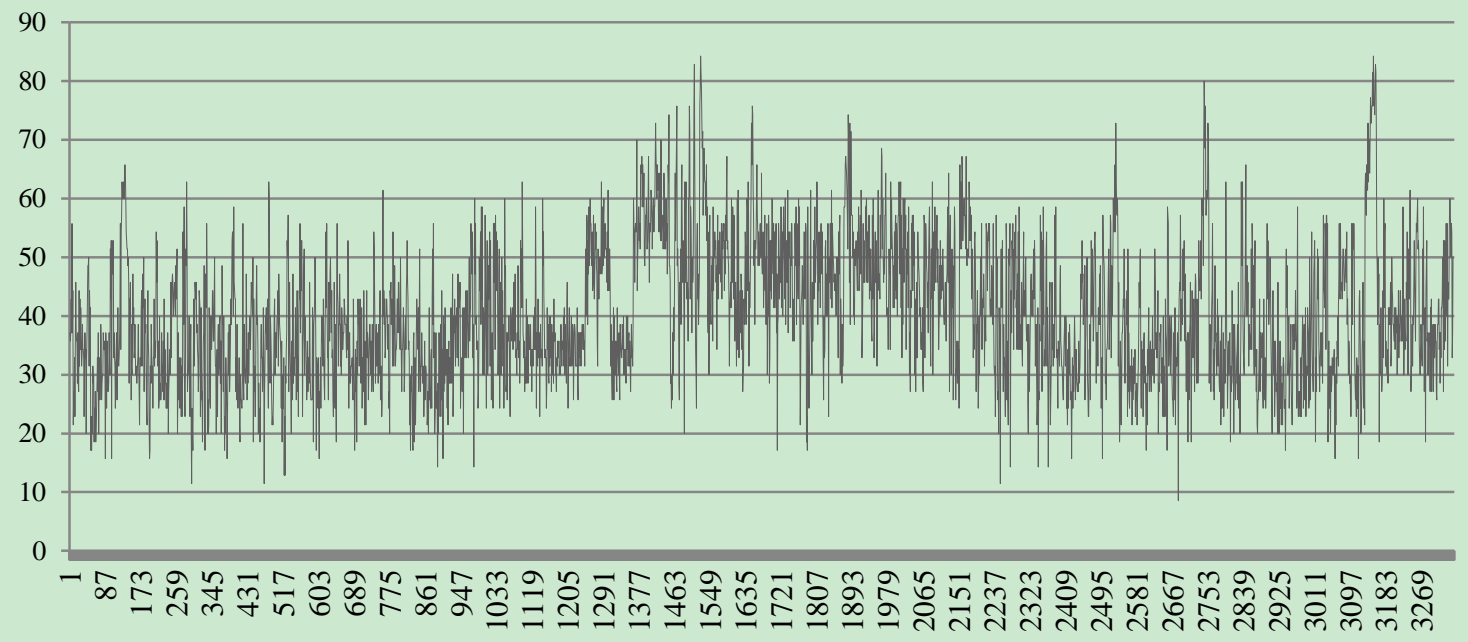

$\mathrm{C}+\mathrm{G}$ percentage

Base pair(KBp)

Fig. 1. CG percentage of NT_025215.4.

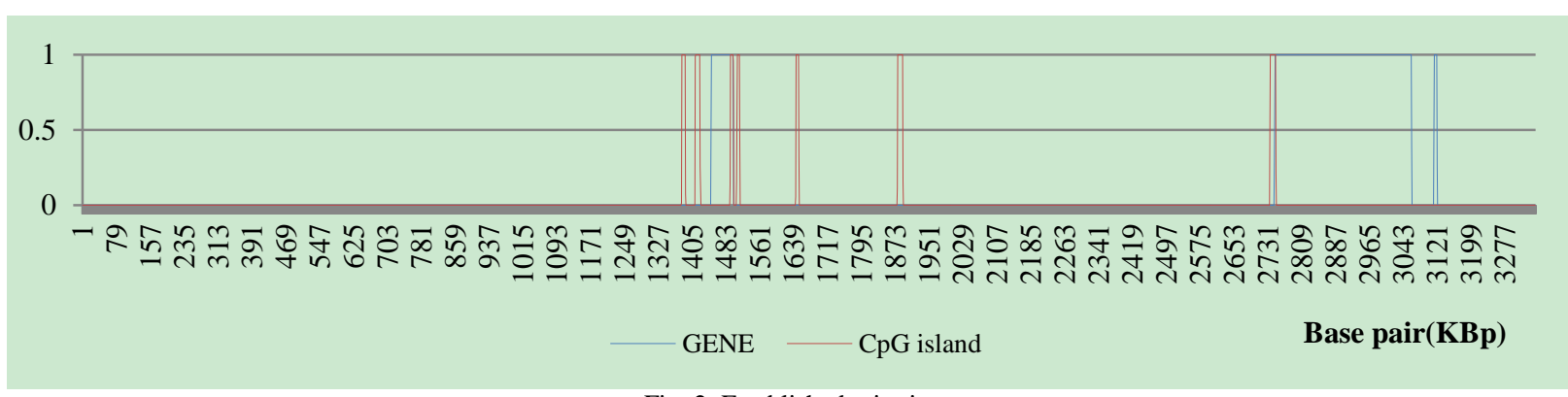

Fig. 2. Established criteria. 


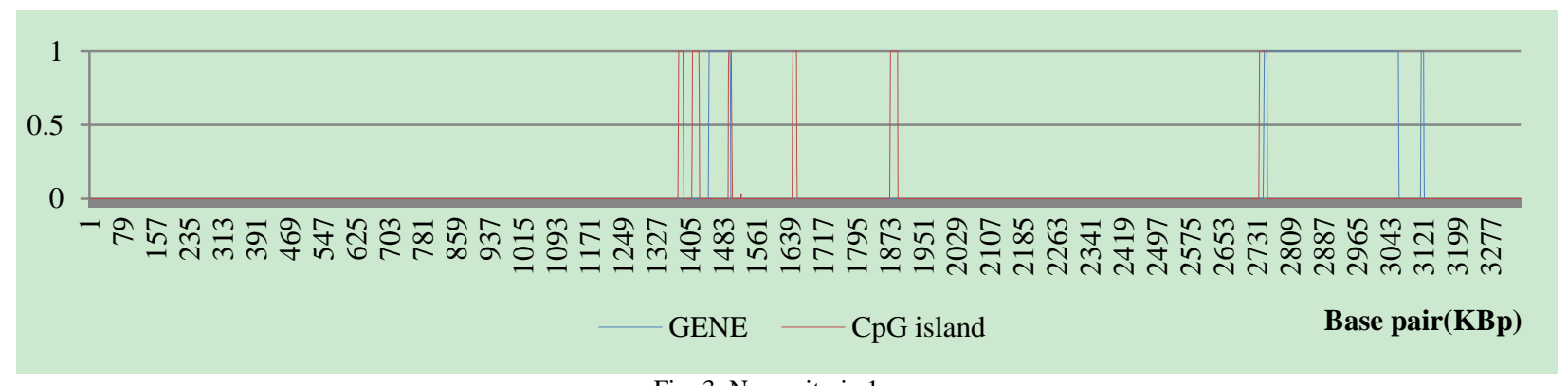

Fig. 3. New criteria 1.

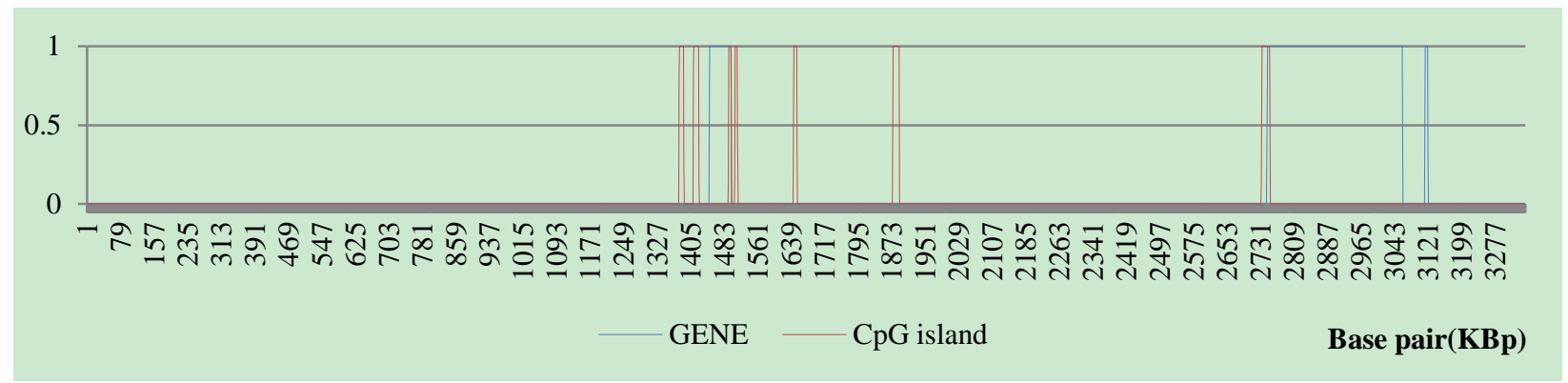

Fig. 4. New criteria 2.

\section{CONCLUSION}

Our study have proved two newly suggested criteria's possibility of being promoted in future studies, as both criteria have shown significant ability of finding genemarker $\mathrm{CpG}$ islands. Higher $\mathrm{G}+\mathrm{C}$ content was proved to improve gene-relation rate, compared to established criteria. Moreover, by comparing our two newly suggested criteria, we found out that the higher o/e ratio is, the higher promoter-relation rate their $\mathrm{CpG}$ islands show. This might be explained by exclusion of Alu repeats, and further research seems to be needed.

We could reach to conclusion that gene location and $\mathrm{CpG}$ island position recorded in some tendency. If CG portion is higher than the others, the higher possibility of $\mathrm{CpG}$ islands positioning, and gene positioning either. Also, $\mathrm{CpG}$ island location and Gene location usually overlapped, as we can assure the tendency to be positive.

\section{ACKNOWLEDGMENT}

Special thanks to Taeseon Yoon, who invited all of us into the world of bioinformatics.

\section{REFERENCES}

[1] A. P. Bird, "DNA methylation patterns and epigenetic memory," Genes Dev., vol. 16, pp. 6-21, 2002.

[2] M. Scherf, A. Klingenhoff, and T. Werner, "Highly specific localization of promoter regions in large genomic sequences by PromoterInspector: a novel context analysis approach," J. Mol. Biol., vol. 297, pp. 599-606, 2000.

[3] M. Gardiner-Garden and M. Frommer, " $\mathrm{CpG}$ islands in vertebrate genomes," J. Mol. Biol., vol. 196, pp. 261-282, 1987.

[4] D. Takai and P. A. Jones, "Comprehensive analysis of CpG islands in human chromosomes 21 and 22," Proc. Natl Acad. Sci. , USA, vol. 99, pp. 3740-3745, 2002.

[5] F. Larsen, G. Gundersen, R. Lopez, and H. Prydz, "CpG islands as gene markers in the human genome," Genomics, vol. 13, pp. 1095-1107, 1992.

[6] Y. Wang and F. C. C. Leung, "An evaluation of new criteria for $\mathrm{CpG}$ islands in the human genome as gene markers," Bioinformatics, vol. 20, pp. 1170-1177, 2004
[7] F. Antequera and A. Bird, "Number ofCpGislands and genes in human and mouse," Proc. Natl Acad. Sci., USA, vol. 90, pp. 11995-11999, 1993.

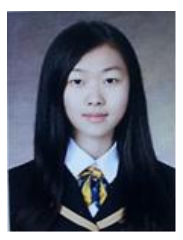

Seonghui Yu was born in 1996. She is currently a student in science major of hankuk academy of foreign studies. She is interested in bio-science and gene analysis associated with clinical psychology which detect human behavior pattern.

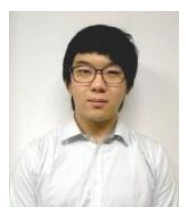

Yeonho Jung was born in 1996. He is currently a student in science major of hankuk academy of foreign studies. He is currently interested in bio-science and modeling process to find most efficient way of analyzing and solving given problems.

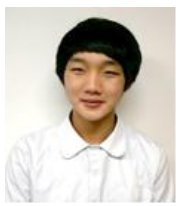

Junhyung Bae was born in 1996. He is currently a student in science major of hankuk academy of foreign studies. He is interested in bio-science and applying bio-infomatics to analyzing gene and finding new facts about human genes.

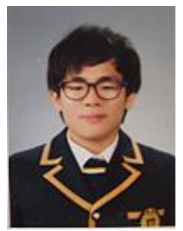

Jongjun Lee was born in 1996. He is currently a student in science major of hankuk academy of foreign studies. He is mostly interested in applying bioinformatics and gene information to basic medical research.

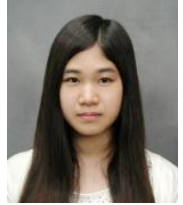

Haewon Kim was born in 1996. She is currently a student in science major of Hankuk Academy of Foreign Studies. She is interested in bio-science and analyzing genetic facts or phenomenon related to humans 